\title{
Comparative study on dynamic mathematical modeling and simulation of coastal sandcastle foundations
}

\author{
Jiarui $\mathrm{Cao}^{1}$, and Wenyue $\mathrm{Zhao}^{2, *}$ \\ ${ }^{1}$ School of Management and Engineering, Nanjing University, 22 Hankou Road, Nanjing, China \\ ${ }^{2}$ School of Business, Nanjing University, 22 Hankou Road, Nanjing, China
}

\begin{abstract}
How to make a sandcastle with good stability is an interesting modeling problem, the core of which is how to design the shape of its foundation and select appropriate material so that it can fully resist wave erosion. First, we design the Foundation Shape Optimization (FSO) Model to obtain the geometric shape that last longest. By pre-screening, we preliminarily select the circular truncated cone. Then we analyse the impact force of the sea waves, list the constraint conditions and calculate the optimal parameters for it. Second, the water-to-sand mixture is identified by microcosmic Normalized Extremum Model. Before getting results, the Ideal Sand Particles Plane (ISPP) Model helps us figure out the equivalent relationship among three functions. Finally, we get the objective function through normalization and work out the best proportion (13.14\%) with the optimal sub-humid angle through Genetic Algorithm (GA).
\end{abstract}

\section{Background}

Each year, various sandcastle contests are held all over the world, especially in America. However, successive waves continuously wash over the elaborately built sandcastles.

The sea wave is a wave of the ocean water produced by the wind. When the wave reaches the coast or the shallow water, its depth becomes shallow, length becomes short, height becomes large, and direction is refracted, due to the influence of terrain. The change in the waveform, along with the friction on the seafloor, slows the moving speed of the nearshore wave [1]. So we normally see waves slowly washing over sandcastles by the seashore, which can be seen as the horizontal force.

As for the fine sand, it is easy to be softened and eroded by the waves. In addition to the gravity itself, raindrop splashing and shoreline erosion could wash away or even soak the sand nearby, which would ultimately cause the sandcastles to collapse. To protect the threedimensional works of art, a firm foundation is badly needed. The foundation should not only be strong, but also higher than the general height of the waves, which is commonly less than 0.1 meters [1]. Meanwhile, the water-to-sand proportion has a decisive influence on the stability of the foundation [2]. Therefore, we take multiple factors into consideration, including the foundation shape and the water-to-sand mixture proportion.

* Corresponding author: zwy1733752642@,163.com 


\section{Foundation Shape Optimization (FSO) model}

Considering its function and the effect of waves, we choose a truncated cone for the foundation instead of a prismoid. In this section, the shape of the truncated cone is discussed in detail, and its optimal values of the parameters are obtained.

\subsection{Nonlinear programming constraint analysis}

\subsubsection{Equivalent Impact Force Field (EIFF) model}

To specify and visualize the forces on the sandcastles, we only consider the main impact of the sea waves on the sandcastle foundation. We neglect the force caused by the retreating of the waves because it is far smaller. Moreover, we perceive that the foundation could not hinder the movement of the waves since the waves are much bigger than the sandcastles.

To make it simple, the average impact force of the waves on the sandcastle foundation could be seen as a force with definite direction, uniform distribution, and continuous action. As a result, we regard the average force as an Impact Force Field $\mathbf{E}_{\mathbf{s}}$, which is an irrotational field. To target to the optimization objective, only the force on the front surface of the foundation will be considered. And the force $\mathbf{F}$ in the field points horizontally in the direction towards the shore. This is shown in Figure 1.

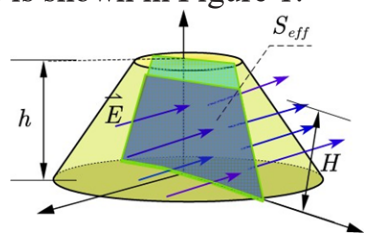

Fig. 1. The sandcastle foundation in the impact force field $\mathbf{E}_{\mathbf{s}}$.

Furthermore, for the sandcastle foundation in $\mathbf{E}_{\mathbf{s}}$, the force on its front surface is proportional to its effective area, $S_{\text {eff. }} S_{\text {eff }}$ is the projected area perpendicular to the field line. Apparently, $S_{\text {eff }}$ can be calculated with the formula of the trapezoidal area.

Consequently, the smaller the $S_{\text {effs }}$ is, the smaller the $\mathbf{F}$ will be. So our goal is to find the circular truncated cone with the minimum $S_{\text {effs, }}$, when its volume is constant. This would be the foundation lasting the longest. Meanwhile, the side area of the foundation $S_{s a}$ should be the smallest so that least partial of sand would be washed away.

\subsubsection{Sandcastle foundation height constraints model}

To ensure the stability, the height of its foundation should be restricted by certain limiting conditions, such as the upper and lower radius of the foundation $r$ and $R$, the elastic modulus $G$ and the density $\rho$ of the fine sand [3]. Here is the equation of the upper limit.

$$
h_{\text {limit }}=\left(\frac{9 J_{(-1 / 3)}^{2} G R_{\text {equi }}^{2}}{16 \rho g}\right)^{\frac{1}{3}}
$$

$J \approx 1.8663$ is the minimum positive root of Bessel function of the first-order $-1 / 3, g$ is the gravitational acceleration and $R_{\text {equi }}$ is the symbol of the equivalent radius of the circular truncated cone, which will be deduced next. 
To work out $R_{\text {equi }}$, we build a coordinate system shown in Figure 2. $R_{\text {equi }}$ is the radius of the horizontal section at the centre of gravity.

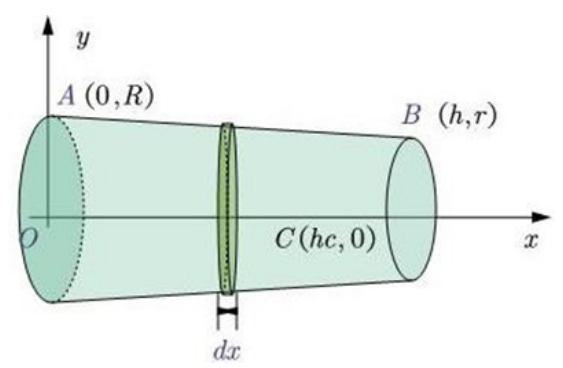

Fig. 2. Diagram of centre of gravity of the truncated cone.

After calculating, we have

$$
R_{\text {equi }}=\frac{R^{2}+2 R r+3 r^{2}}{4\left(R^{2}+R r+r^{2}\right)} R+r
$$

Thus, we should guarantee that the height of the truncated cone $h$ is smaller than $h_{\text {limit }}$, and is a little higher than the average height of the nearshore waves $H$.

Moreover, the lower the center of gravity is, the more stable the three-dimension geometric shape is. So we would like to find the truncated cone with lower center of gravity.

\subsubsection{Three-dimensional slope stability analysis model}

To keep the sand foundation stable, the Slope Angle $\alpha$ should also be restricted to a certain range. We suggest to use the approximate range of the Maximum Natural Slop Angle $\theta$ of the foundation.

The Maximum Natural Slope Angle $\theta$ is the maximum slope angle for the sand foundation to keep stable when the fine sand is piled up [4]. While the Internal Friction Angle $\varphi$ of sand is the angle between the shear strength line and the abscissa axis (the coordinate axis where the normal stress is located) in the shear strength curve. Since the shear strength of sand conforms to the friction law $\tau=\sigma \tan \varphi$, the friction is between the interior of the same material, called 'Internal Friction'. And $\varphi$ shows the relative ease with which the sand particles slide. The bigger the $\varphi$ is, the harder the sand particles relatively slide [4]. The relationship between the two angles will be demonstrated next.

We first scale the slope in Figure 3 and draw a sliding arc of the sand foundation. By infinitesimal, we divide the sand above the sliding surface into several strips.

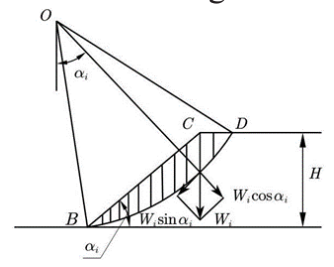

Fig. 3. Microscopic diagram of sliding body forces.

If we symbol the gravity by $W_{i}$ and the length of each sand stripe by $l_{i}$, we could define the Total Moment of Force in both horizontal and vertical directions as follows

$$
\begin{aligned}
& T R=R \sum T_{i}=R \sum W_{i} \sin \alpha_{i} \\
& T^{\prime} R=R \sum N_{i} \tan \varphi_{i}=R \sum W_{i} \cos \alpha_{i} \tan \varphi_{i}
\end{aligned}
$$

When 


$$
F_{s}=\frac{T^{\prime} R}{T R}=\frac{R \sum W_{i} \cos \alpha_{i} \tan \varphi_{i}}{R \sum W_{i} \sin \alpha_{i}}>1
$$

the sand would not slide and the sand foundation would keep stable, which indicate that $\varphi>$ $\alpha$. What's more, we could conclude as $\theta=\varphi$, and we select $20^{\circ}$ for $\theta_{1}$ and $80^{\circ}$ for $\theta_{2}$ [4].

$$
\theta_{1}<\alpha<\theta_{2}
$$

\subsection{Model solution based on Monte Carlo Algorithm (MCA)}

According to the restrictions above, our Nonlinear Programming Model of the minimum area $S$ of the sandcastle foundation can be

$$
\begin{aligned}
\min S & =\alpha_{1} S_{\text {effs }}+\alpha_{2} S_{s a} \\
\text { s.t. } V & =\pi h\left(R^{2}+r^{2}+R r\right) / 3 \\
A X & \leqslant B \\
H & <h<h_{\text {limit }} \\
0 & <r<R
\end{aligned}
$$

where the elastic modulus $G=0.35 \mathrm{MPa}$, the density $\rho=1.7 \times 10^{3} \mathrm{~kg} / \mathrm{m}^{3}$, the gravitational acceleration $g=9.81 \mathrm{~N} / \mathrm{kg}, \theta_{1}=20^{\circ}$ and $\theta_{2}=80^{\circ} . \alpha_{1}$ and $\alpha_{2}$ are weights set to synthetically the effects of the two areas. In addition, $A, B$ and $X$ symbol matrixes

$$
A=\left(\begin{array}{ccc}
-1 & 1 & 0 \\
\tan \theta_{1} & -\tan \theta_{1} & -1 \\
\tan \theta_{2} & -\tan \theta_{2} & 1
\end{array}\right) \quad B=\left(\begin{array}{l}
0 \\
0 \\
0
\end{array}\right) \quad X=\left(\begin{array}{l}
R \\
r \\
h
\end{array}\right)
$$

Through MATLAB programming, we get our optimal circular truncated cone with the minimum $S$, when its volume is constant. The results are $R_{\text {opti }}=3.8799, r_{\text {opti }}=3.2478, h_{\text {opti }}$ $=1.0213$.

Furthermore, as $V$ increasing, $R$ and $r$ increases almost proportionately and the slope angle $\alpha$ keeps steady at about $68^{\circ}$, shown is Figure 4 . This conforms well to reality, especially the slope angle, because people prefer to mode the foundation out of a bracket.

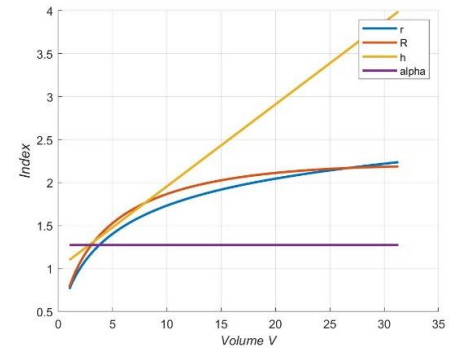

Fig. 4. Relationship between truncated cone parameters and volume change.

\section{Normalized extremum model on unsaturated soil mechanics}

\subsection{Theory of Ideal Sand Particles Plane (ISPP) model}

To visualize the microscopic relationship between water and sand, we design an Ideal Sand Particles Plane Model, which helps to calculate the volume ratio of water and sand [2]. 


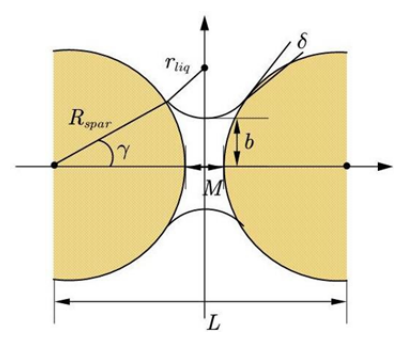

Fig. 5. Diagram of ideal sand particles plane model.

By the Pythagorean Theorem, we have

$$
\left\{\begin{array}{l}
\left(x-a-R_{s p a r}\right)^{2}+y^{2}=R_{s p a r}^{2} \\
x^{2}+\left(y-r_{\text {liq }}-b\right)^{2}=r_{\text {liq }}^{2} \\
y^{\prime}=-\frac{x}{y-r_{\text {liq }}-b}=\tan \left(\frac{\pi}{2}-\delta-\gamma\right) \\
r_{\text {liq }}=\frac{R_{\text {spar }}(1-\cos \gamma)}{\cos \gamma} \\
b=\frac{R_{\text {apar }}(\sin \gamma+\cos \gamma-1)}{\cos \gamma}
\end{array}\right.
$$

If we consider that the meniscus rotates in an arc around the axes of the two ball centres, we have the volume of the liquid bridge between two sand particles $V_{\text {liq }}$.

$$
\begin{aligned}
V_{\text {liq }}= & 2 \pi \int_{0}^{a-R_{\text {spar }}-R_{\text {spar }} \cos \gamma}\left(\sqrt{r^{2}-x^{2}}+r_{\text {liq }}+b\right)^{2} d x \\
& -2 \pi \int_{0}^{a-R_{\text {spar }}-R_{\text {sar }} \cos \gamma}\left[R_{\text {spar }}^{2}-\left(x-a-R_{\text {spar }}\right)^{2}\right] d x
\end{aligned}
$$

However, it is not easy to accurately obtain the relationship between $V_{l i q}$ and $\gamma$. So we use the Fisher Empirical Formula to facilitate the calculation of $V_{l i q}$.

$$
V_{\text {liq }}=2 \pi R_{\text {spar }}^{3}(\sec \gamma-1)^{2}[1-(\pi / 2-\gamma) \tan \gamma]
$$

\subsection{Normalization of three functions and Genetic Algorithm (GA) solution}

Since the volume of the liquid bridge $V_{\text {liq }}$ is a function of the sub-humid angle $\gamma$ and the volume of each sand particle can be easily worked out, we expect to obtain the best subhumid angle so as to calculate the best volume proportion of water to sand.

Plenty of researchers have studied the relationship between soil and water in the field of unsaturated soil mechanics. Due to the similarity between soil and sand, we choose three important functions to discuss their influence on the solidity of the sandcastle foundation, including surface tension, capillary cohesion and dry density.

After normalizing the three functions, we set weights for each of them and get our objective function. Then we use Genetic Algorithm with MATLAB programming to calculate the maximum value of the objective function and get the optimal $\gamma_{\text {opti }}$ and the best water-to-sand mixture proportion $P_{\text {opti }} \approx 13.14 \%$ with

$$
P=\frac{V_{\text {water }}}{V_{\text {sand }}}=\frac{N V_{\text {liq }} N_{c}}{V_{\text {spar }} N}=\frac{3 V_{\text {liq }} N_{c}}{4 \pi R_{\text {spar }}^{3}}
$$




\section{References}

1. Limin S. Wave formation and wave level. Proceedings of the symposium on new experiences and new technologies in preventing shipwreck accidents (vol.2). Maritime ship steering com-mittee, China institute of navigation, 2004: 66-71.

2. Chang L., Junying C., Zhitao Z., Hongyang C., Yaohui C. Experimental Study and Ideal Model Analysis of the Relationship between Sand Water Repellency and Moisture Content. Water-saving irrigation, 1007-4929(2017): 08-0013-04.

3. Maryam P., Mehdi H., Peder M., Daniel B. How to construct the perfect sandcastle. Scientific Reports, 2012.

4. Zhijie F., Jianjun Q., Huan Z. The Relationship between the Internal Friction Angle of Sand Soil and Grain Size, Water Content and Natural Slope Angle. China Desert, 2015, 35(2): 301-305. 